МИНИСТЕРСТВО ОБРАЗОВАНИЯ И НАУКИ РОССИЙСКОЙ ФЕДЕРАЦИИ

ТОМСКИЙ ГОСУДАРСТВЕННЫЙ УНИВЕРСИТЕТ

\title{
МАТЕРИАЛЫ
}

VIII Международной молодежной научной конференции

«МАТЕМАТИЧЕСКОЕ

И ПРОГРАММНОЕ ОБЕСПЕЧЕНИЕ

ИНФОРМАЦИОННЫХ, ТЕХНИЧЕСКИХ

И ЭКОНОМИЧЕСКИХ СИСТЕМ»

Томск, 26-30 мая 2021 г.

Под общей редакиией И.С. Шмырина

Томск

Издательство Томского государственного университета 2021 
Маркова с непрерывным временем. Для описания функционирования модели была составлена система дифференциально-конечноразностных уравнений Колмогорова (1). Как результат, с помощью метода частичных характеристических функций и модификации метода асимптотического анализа была получена формула (4), являющаяся асимптотическим приближением характеристической функции числа событий, наступивших в выходящем потоке системы M/M/1. Применив к полученной формуле (4) обратное преобразование Фурье, было получено распределение вероятностей числа событий, наступивших в выходящем потоке за время $t$, формула (5).

DOI: $10.17223 / 978-5-907442-42-9-2021-27$

\section{ЗАДАЧА МАRL ДЛЯ СВЕТОФОРА НА ПЕРЕКРЁСТКЕ}

Тисленко Т.И.

Сибирский федеральный университет timtisko@mail.ru

\section{Введение}

В Красноярске с каждым годом растет количество желающих стать автомобилистом. По данным агентства «Автостат» на 1 января 2020 г. Красноярский край оказался на 12 месте в топ-20 регионов по объему автомобильного парка в России. С количеством автомобилистов увеличивается и время, которое проводится в пробках. Таким образом, проблема оптимизации сети светофоров с целью уменьшения задержек трафика автомобилей является актуальной и востребованной в современных мегаполисах. Решение проблемы - согласование действий сети светофоров по управлению процессом выбора задержки сигнала. Внедрение адаптивных систем светофоров в городскую транспортную сеть может его уменьшить.

На данный момент существует множество адаптивных систем управления трафиком. Например, модель Split Cycle Offset Optimization Technique (SCOOT) в Великобритании [1]. При ее использовании транспортные средства обнаруживаются по мере приближения к перекрестку задолго до стоп-линии, затем с нескольких перекрестков сигналы об обнаружении передаются в центральную систему, которая моделирует поток транспорта в этом районе и производит адаптацию фаз светофора в соответствии с трафиком, тем самым сводя к минимуму ненужные зеленые фазы. Также к централизованному типу координации можно отнести систему Traffic-responsive Urban Control (TUC) в Греции $[1,2]$. Использование центральных систем координации в случае большого количества перекрестков ресурсозатратно, поэтому в качестве альтернативы возникли распределенные модели [1]: Sydney Coordinated Adaptive Traffic System (SCATS) в Австралии, Dynamic Programming Algorithm (PRODYN) и Real-time Hierarchical Optimized Distributed Effective System (RHODES). Приведенные системы оперируют количеством транспортных средств, проходящих на определенном отрезке полосы дороги, и поэтому их можно отнести к моделям, основанным на плотности потока.

Как альтернативный способ координации можно рассмотреть модели, основанные на марковских процессах принятия решений, которые могут не оперировать интенсивностью потока. В качестве математической модели сети светофоров в работе рассматривается управляемый марковский процесс с конечным числом действий и состояний $[3,4]$. Каждый агент (светофор) не располагает ресурсами и решает задачу целесообразности выбора того или иного сигнала. Среда - перекресток с машинами, где на отрезках дорог за 100 м до стоп-линий засекается время. Состояние среды отражает активность фазы светофора. Тогда проблема оптимизации сводится к задаче MARL (Multiagent Reinforcement Learning) [4]. Как правило, обучение с подкреплением используется для одного агента в среде, чтобы максимизировать его долгосрочную награду. Модель среды - марковский процесс принятия решения [5]. В работе исследуется 
модель $Q$-обучения одного агента (светофора) в стационарной среде [6]. $Q$-обучение сводится к оптимальному сопоставлению агентом действия $a$ состоянию окружающей среды $s$ на основе кумулятивных наград $r(s, a)$. Предложенная математическая модель процесса выбора фазы светофора учитывает текущее расположение светофоров и их загрузки и позволяет сформулировать оптимизационные задачи, целью которых является минимизация задержки трафика автомобилей. Отметим, что структура мультиагентной системы, включающая в себя единственного агента - светофор, обеспечивает наиболее эффективное распараллеливание всей задачи на подзадачи, которые будут решены агентом.

\section{1. Задача MARL для одного светофора}

Пусть светофор - это агент, не располагающий ресурсами. В качестве среды будем рассматривать перекресток с машинами, где на отрезках дорог за 100 м до стоп-линий засекается время (рис. 1).

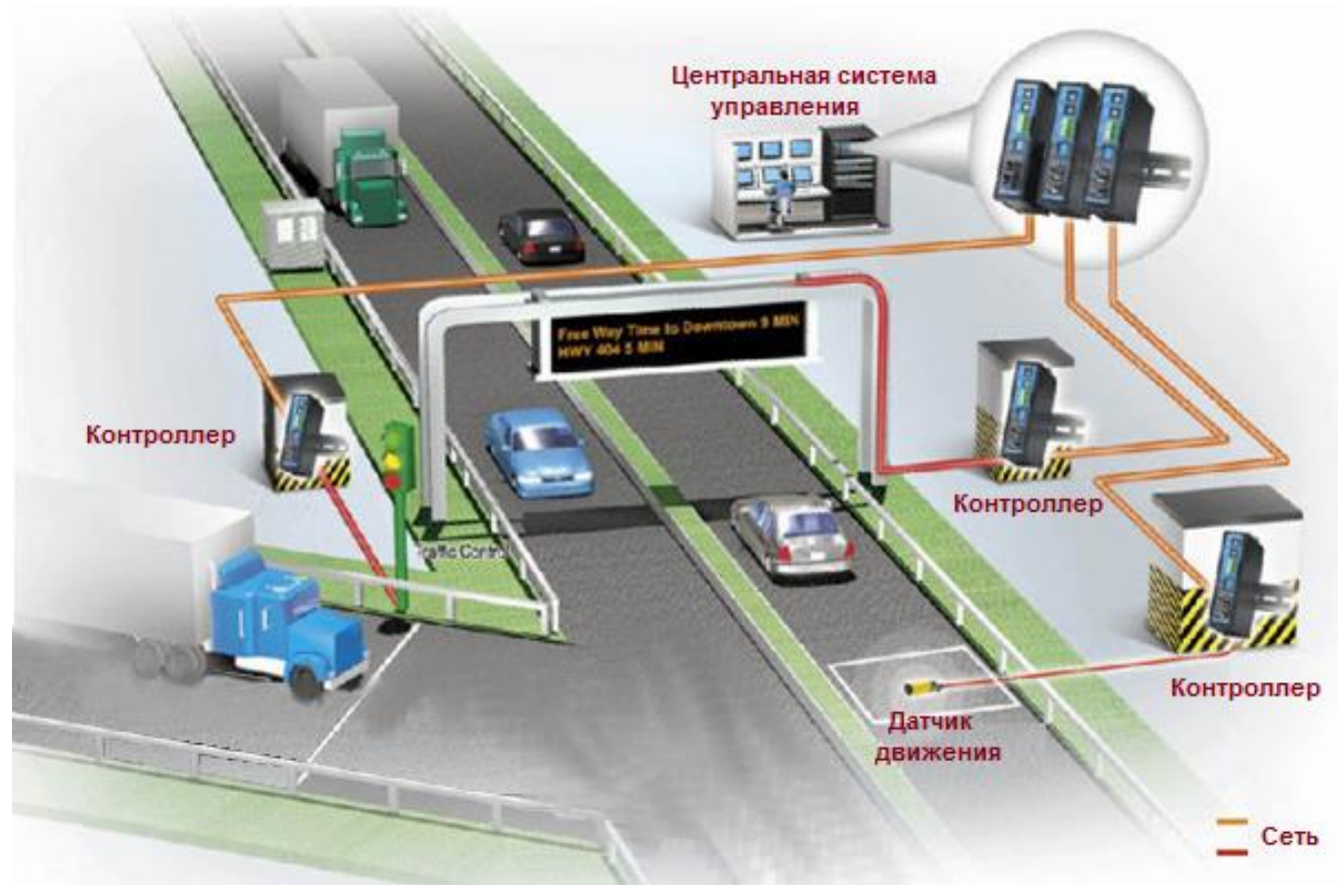

Рис. 1. Визуальная интерпретация модели

Считаем, что состояние среды отражает активность фазы светофора. Обозначим $S=\left\{s_{0}=\right.$ «активна фаза $0 », s_{1}=$ «активна фаза $\left.1 »\right\}-$ пространство состояний агента, а множество решений агента как $A=\left\{a_{0}=\right.$ «оставить фазу», $a_{1}=$ «сменить фазу» $\}$. Диаграмма переходов для светофора представлена на рис. 2.

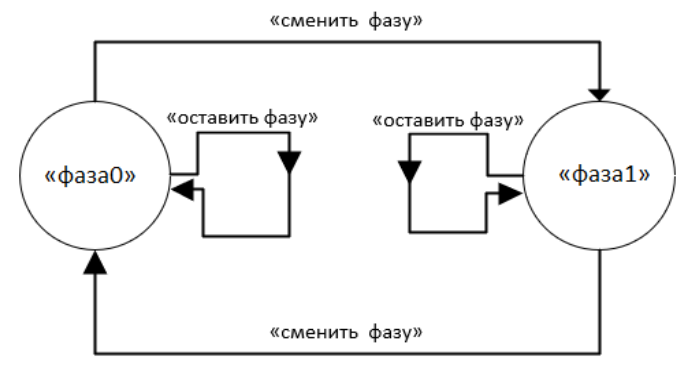

Рис. 2. Диаграмма переходов для задачи управления светофором 
Пусть в момент времени $t_{k}$ активна фаза светофора $S_{k}$. Под задержкой на фазе $S_{k}$ будем понимать суммарное засечённое время всех машин, проходящих через отрезок дороги. Задержка на фазе s после действия $a$ есть функция вознаграждения $r(s, a)$. Будем считать, что функция вознаграждения всецело определяется текущим состоянием, выбранной стратегией и состоянием, в которое перейдет процесс на следующем шаге. Далее предполагаем, что вероятность перехода $p\left(S_{i}, A_{k} ; S_{j}\right)$ системы из состояния $S_{i}$ при выборе решения $A_{k}$ в состояние $S_{j}$ полностью определяется состоянием, в которое переходит процесс.

Функция суммарных доходов от оптимальной политики в состоянии $s$ в общем виде задается формулой [5]:

$$
V^{*}(s)=\max _{A(\bullet)} \sum_{t=0}^{\infty} \gamma^{t} r\left(s_{t}, a_{t}\right),
$$

где $\gamma$ - это коэффициент переоценки, а $A(\bullet)$ - управление.

Известно [4], что уравнение Вальда-Беллмана для управляемого марковского процесса с конечным числом действий и состояний в общем виде имеет вид

$$
V^{*}(s)=\max _{a \in A} \sum_{s^{\prime} \in S} p\left(s, a ; s^{\prime}\right)\left(r\left(s, a ; s^{\prime}\right)+\gamma V^{*}\left(s^{\prime}\right)\right) .
$$

C учётом (1) и (2) задача MARL для одного светофора примет следующий вид.

\section{Задача MARL для одного светофора}

Требуется найти такое управление $A(\bullet)$, которое доставляет максимум функции

$$
\begin{array}{r}
V^{*}(s)=\max \left\{p\left(s, A_{0} ; S_{0}\right)\left(r\left(s, A_{0}\right)+\gamma V^{*}\left(S_{0}\right)\right)+p\left(s, A_{0} ; S_{1}\right)\left(r\left(s, A_{0}\right)+\gamma V^{*}\left(S_{1}\right)\right) ;\right. \\
\left.p\left(s, A_{1} ; S_{0}\right)\left(r\left(s, A_{1}\right)+\gamma V^{*}\left(S_{0}\right)\right)+p\left(s, A_{1} ; S_{1}\right)\left(r\left(s, A_{1}\right)+\gamma V^{*}\left(S_{1}\right)\right)\right\},
\end{array}
$$

где функция $V^{*}(s)$ представима в виде $V^{*}(s)=\max _{a \in A} Q(s, a)=\max _{a \in A} Q_{t}(s, a)$, а $Q(s, a)$ есть функция

$$
Q(s, a)=\sum_{s^{\prime} \in S} p\left(s, a ; s^{\prime}\right)\left(r\left(s, a ; s^{\prime}\right)+\gamma V^{*}\left(s^{\prime}\right)\right)=\sum_{s^{\prime} \in S} p\left(s, a ; s^{\prime}\right)\left(r\left(s, a ; s^{\prime}\right)+\gamma \max _{a^{\prime} \in A} Q\left(s^{\prime}, a^{\prime}\right)\right) .
$$

Решение задачи MARL для одного светофора базируется на идее $Q$-обучения, которая заключается в оценке невычислимой правой части [4]:

$$
Q_{t+1}(s, a)=Q_{t}(s, a)+\alpha_{t}(s, a)\left(r(s, a)+\gamma \max _{a^{\prime} \in A} Q_{t}\left(s^{\prime}, a^{\prime}\right)-Q_{t}(s, a)\right),
$$

где $s^{\prime}-$ положение процесса на шаге $t+1$, если на шаге $t$ в состоянии $s$ было выбрано действие $a ; \alpha-$ коэффициент скидки.

Если на шаге $t$ итерационный процесс находился в состоянии $s$ и было выбрано действие $a$, то в (3) полагаем $0 \leq \alpha_{t} \leq 1$, иначе $\alpha_{t}=0$.

Последовательность векторов $Q=\{Q(s, a)\}_{s \in S, a \in A}$ можно записать итеративно в виде $Q_{t+1}=B\left(Q_{t}\right)$, где $B: \square_{\infty}^{|A++| S \mid} \rightarrow \square_{\infty}^{|A|+|S|}$ является сжимающим отображением [4]. Действительно, 


$$
\begin{aligned}
\rho\left(B Q_{1}, B Q_{2}\right)= & \max _{a \in A, s \in S} \mid \sum_{s^{\prime} \in S} p\left(s, a ; s^{\prime}\right)\left(r\left(s, a ; s^{\prime}\right)+\gamma \max _{a^{\prime} \in A} Q_{1}\left(s^{\prime}, a^{\prime}\right)\right)- \\
& -\sum_{s^{\prime} \in S} p\left(s, a ; s^{\prime}\right)\left(r\left(s, a ; s^{\prime}\right)+\gamma \max _{a^{\prime} \in A} Q_{2}\left(s^{\prime}, a^{\prime}\right)\right) \mid= \\
= & \max _{a \in A, s \in S}\left|\sum_{s^{\prime} \in S} p\left(s, a ; s^{\prime}\right) \gamma\left(\max _{a^{\prime} \in A} Q_{1}\left(s^{\prime}, a^{\prime}\right)-\max _{a^{\prime} \in A} Q_{2}\left(s^{\prime}, a^{\prime}\right)\right)\right| \leq \\
\leq & \gamma \max _{a \in A, s \in S} \sum_{s^{\prime} \in S} p\left(s, a ; s^{\prime}\right)\left|\max _{a^{\prime} \in A} Q_{1}\left(s^{\prime}, a^{\prime}\right)-\max _{a^{\prime} \in A} Q_{2}\left(s^{\prime}, a^{\prime}\right)\right| \leq \\
\leq & \gamma \max _{s^{\prime} \in S}\left|\max _{a^{\prime} \in A}\left(Q_{1}\left(s^{\prime}, a^{\prime}\right)-Q_{2}\left(s^{\prime}, a^{\prime}\right)\right)\right| \leq \\
\leq & \gamma \max _{a^{\prime} \in A, s^{\prime} \in S}\left|Q_{1}\left(s^{\prime}, a^{\prime}\right)-Q_{2}\left(s^{\prime}, a^{\prime}\right)\right|=\gamma \max _{a \in A, s \in S}\left|Q_{1}(s, a)-Q_{2}(s, a)\right|=\gamma \rho\left(Q_{1}, Q_{2}\right),
\end{aligned}
$$

где $0<\gamma<1, \rho(\bullet, \bullet)-$ метрика в $\square_{\infty}^{|A|+|S|}$.

Полученная последовательность $\left\{Q_{t}\right\}$ представляет собой приближенные решения уравнения $B Q=Q$. Таким образом, начиная с определенного момента времени вычислять значение функции $Q$ не требуется, и эффективная оценка точности такого приближенного решения рассчитывается по формуле

$$
\rho\left(Q_{t_{n}}, Q_{t_{0}}\right)=\rho\left(B^{n} Q, Q\right) \leq \frac{\gamma^{n} \rho(B Q, Q)}{1-\gamma} .
$$

Теперь можем сформулировать критерий сходимости процесса $\left\{Q_{t}\right\}$ [4].

\section{Критерий сходимости}

Если стратегия $A(\bullet)$ приводит к тому, что каждая пара $(s, a)$ бесконечное число раз встречается, то из условия сжимаемости $B$ при $\sum_{t=0}^{\infty} \alpha_{t}=\infty, \sum_{t=0}^{\infty} \alpha_{t}^{2} \leq \infty$ следует сходимость процесса $\left\{Q_{t}\right\}$ с оценкой (4).

С учётом формул (3)-(5) будем искать оптимальное управление в виде $a_{t}=\arg \max _{a^{\prime} \in A} Q_{t}\left(s, a^{\prime}\right)$. Оптимальное управление $a_{t}$ является решением задачи MARL для одного светофора, т.е. доставляет максимум функции суммарных доходов $V_{t}^{*}=\max _{a \in A} Q_{t}(s, a)$.

\section{2. Вычислительные эксперименты}

Для исследования представленной модели была разработана программа имитационного моделирования в системе AnyLogic и проведены серии вычислительных экспериментов. Эксперименты проводились на ПК с процессором Intel® Core $^{\mathrm{TM}}$ i7-10510U

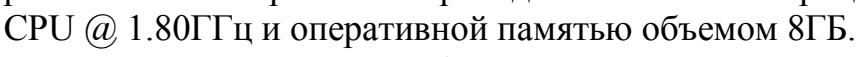

Целью экспериментов было сравнение времени задержки машин в модели системы управления светофоров, длительность фаз р0, p1 которой получена перебором (рис. 3), и управляемой марковским процессом (рис. 4), в системе Anylogic. Имитационное моделирование процесса управления светофором проводилось с учётом следующих условий (рис. 3, 4):

1) машины прибывают на перекресток с каждого из трех направлений: carSource, carSource1, carSource2, с интенсивностью 1000 в час,

2) коэффициенты скидки $\alpha$ (alfa) и переоценки $\gamma$ (gamma) подобраны эмпирическим путём, 
3) дискретное время period составляет 5 секунд.

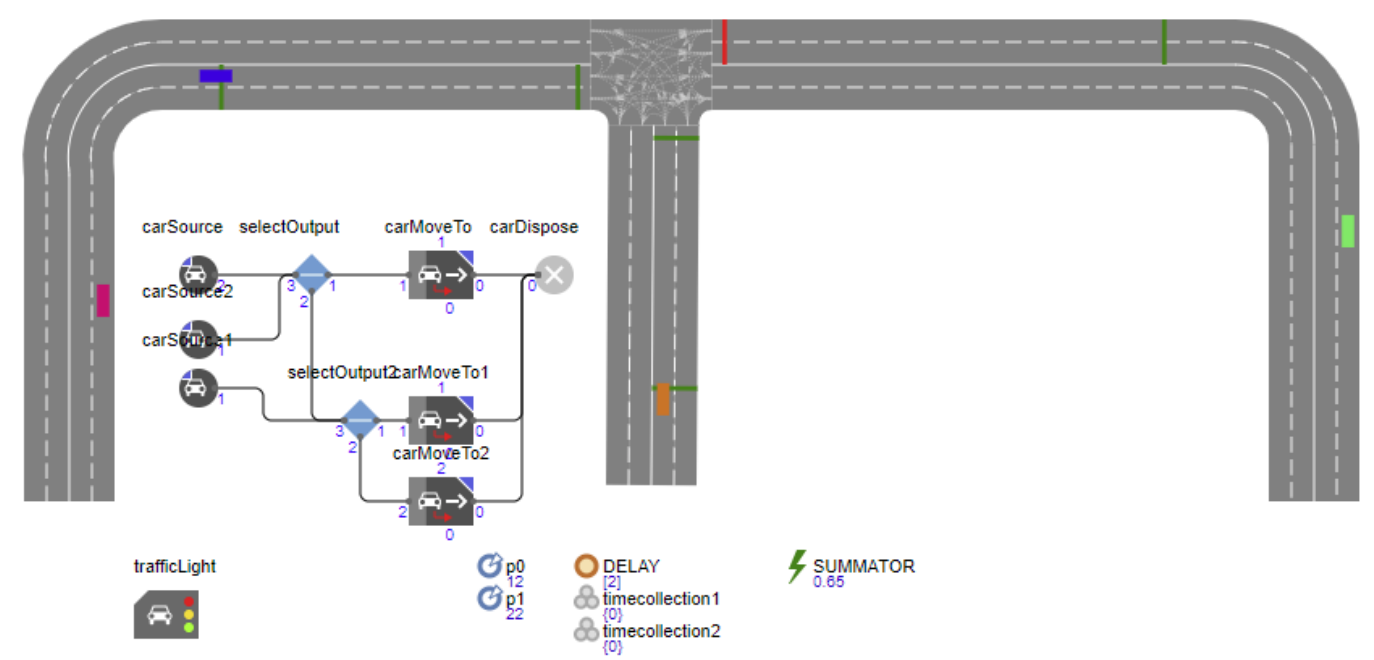

Рис. 3. Модель, длительность фаз которой получена перебором

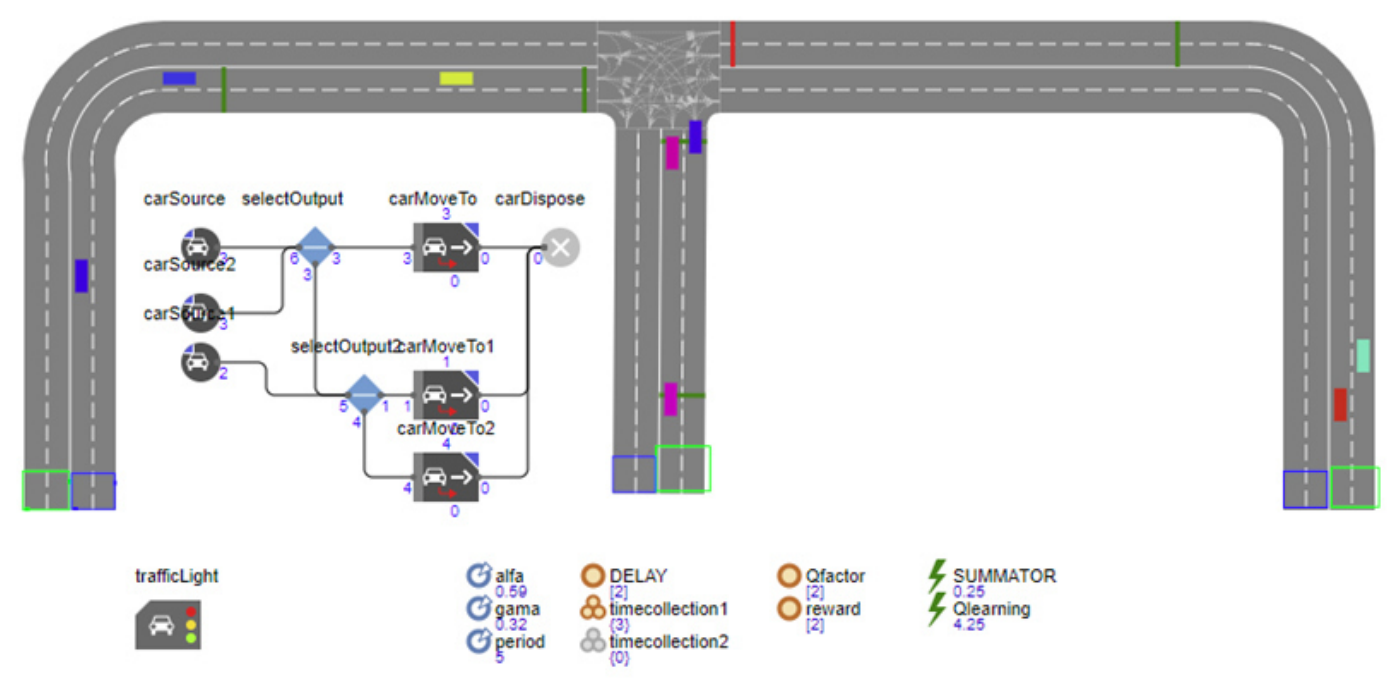

Рис. 4. Модель, управляемая марковским процессом

При имитационном моделировании машины создаются на одной из позиций, отмеченных зеленым цветом, и перемещаются в направлении позиций, отмеченных синим, после чего удаляются (рис. 4). При пересечении полосы, на расстоянии 100 метров до стоп-линии, пары, состоящие из указателей на объект машины и текущего времени модели, добавляются в одну из коллекций timecollection, timecollection1, timecollection2. Далее машины удаляются из коллекции при проезде через перекресток. В течении периода времени 1 секунда вызывается событие SUMMATOR, прибавляющее суммарное время задержки машин, находящихся в коллекции, к текущей задержке DELAY. B течении периода времени period вызывается событие Qlearning, реализующее решение задачи MARL. На основе события Qlearning принимается решение об остановке или продлении фазы светофора.

Результаты и выводы: модель, управляемая марковским процессом, показала уменьшение суммарной задержки в 1.5 раза по сравнению с системой управления светофором, длительность фаз которой подобрана перебором от 5 секунд до 30 секунд с 
шагом 1. Значение вектора всевозможных значений функции $Q(s, a)$ по метрике из $\square_{\infty}^{|A|+|S|}$ сходится (рис. 5).

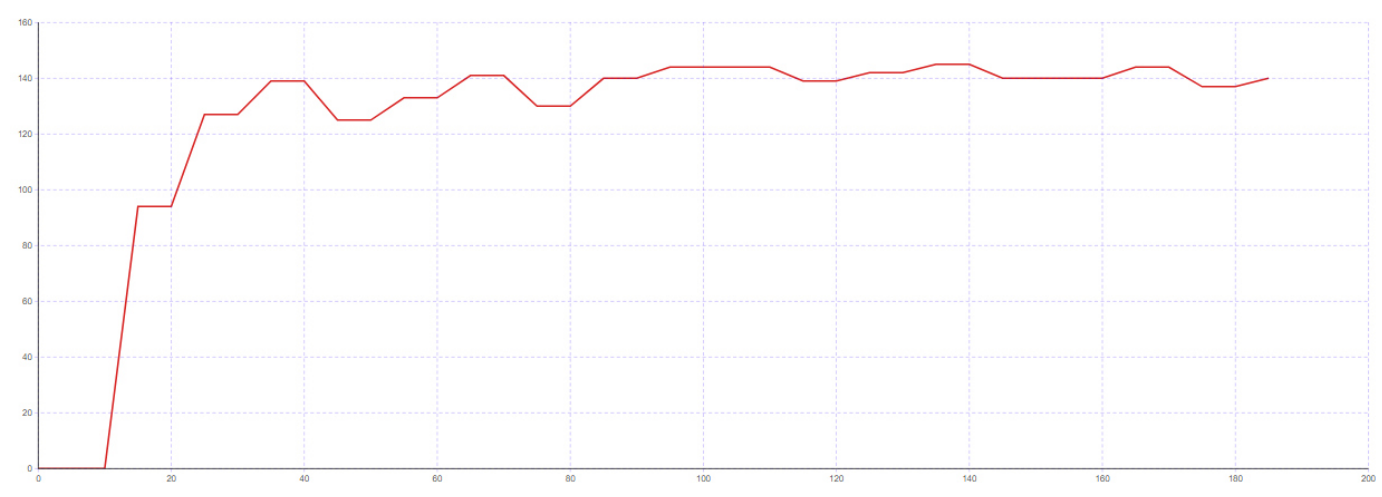

Рис. 5. График сходимости итерационного процесса для модели, управляемой марковским процессом

\section{Заключение}

В работе исследована математическая модель процесса выбора фазы светофора, отличающаяся учетом текущего расположения светофора и его загрузки. Сформулирована задача MARL для одного светофора на перекрестке. Решение задачи минимизации задержки трафика рассматривается как итерационный процесс, для которого получен критерий сходимости. Проведено имитационное моделирование в среде Anylogic 8. В дальнейшем планируется увеличить количество агентов и сравнить результаты с существующими моделями.

\section{ЛИТЕРАТУРА}

1. Goel S., Bush S., Gershenson C. Self-Organization in Traffic Lights: Evolution of Signal Control with Advances in Sensors and Communications ArXiv. - 2017. - abs. 1708.07188.

2. Manolis D., Diakaki C., Papamichail I. et al. Simulation investigations of the coordinated traffic-responsive signal control strategy TUC with actuation at the local junction level // Eur. Transp. Res. Rev. - 2018. - 10. - 25 .

3. El-Tantawy S., Abdulhai B., Abdelgawad H. Multiagent Reinforcement Learning for Integrated Network of Adaptive Traffic Signal Controllers (MARLIN-ATSC): Methodology and Large-Scale Application on Downtown Toronto // IEEE Transactions on Intelligent Transportation Systems. - 2013. V. 14. - No. 3. - P. 1140-1150.

4. Гасников А.В., Горбунов Э.А., Гуз С.А. и др. Лекции по случайным процессам: учебное пособие. Под ред. А.В. Гасникова. - «Москва»: МФТИ, 2019. -285 с.

5. Майн Х., Осаки С. Марковские процессы принятия решений. - Главная редакция физикоматематической литературы издательства «Наука», 1977. - 176 с.

6. Sandholm T.W. Contract Types for Satisficing Task Allocation: I Theoretical Results // AAAI Spring Symposium Series: Satisficing Models. - 1998. - P. 68-75. 